\title{
Solvent Effects and Mechanism for a Nucleophilic Aromatic Substitution from QM/MM Simulations
}

\author{
Orlando Acevedo and William L. Jorgensen* \\ Department of Chemistry, Yale University, 225 Prospect Street, \\ New Haven, Connecticut 06520-8107.
}

Supporting Material

Figure S1. Solute-solvent energy pair distribution in methanol........................... 3

Figure S2. Solute-solvent energy pair distribution in acetonitrile...........................4

Figure S3. Solute-solvent energy pair distribution in DMSO ............................5

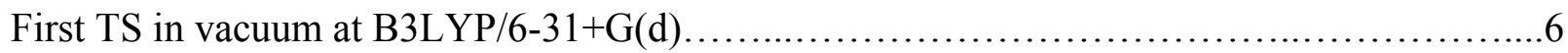

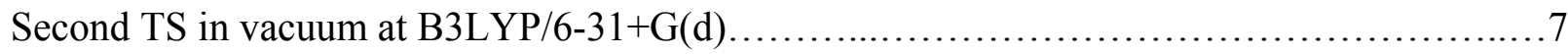

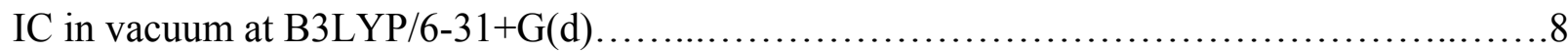

Single point of first TS in PCM (water) at B3LYP/6-311+G(2d,p) .......................

Single point of second TS in PCM (water) at B3LYP/6-311+G(2d,p) $\ldots \ldots \ldots \ldots \ldots \ldots \ldots \ldots \ldots$

Single point of IC in PCM (water) at B3LYP/6-311+G(2d,p) ..........................

Single point of first TS in PCM (methanol) at B3LYP/6-311+G(2d,p)......................10

Single point of second TS in PCM (methanol) at B3LYP/6-311+G(2d,p) .................. 10

Single point of IC in PCM (methanol) at B3LYP/6-311+G(2d,p) ......................... 10

Single point of first TS in PCM (acetonitrile) at B3LYP/6-311+G(2d,p) ...................11

Single point of second TS in PCM (acetonitrile) at B3LYP/6-311+G(2d,p) .................11

Single point of IC in PCM (acetonitrile) at B3LYP/6-311+G(2d,p) ......................11

Single point of first TS in PCM (DMSO) at B3LYP/6-311+G(2d,p) .......................12

Single point of second TS in PCM (DMSO) at B3LYP/6-311+G(2d,p) ................... 12

Single point of IC in PCM (DMSO) at B3LYP/6-311+G(2d,p) .......................... 12 


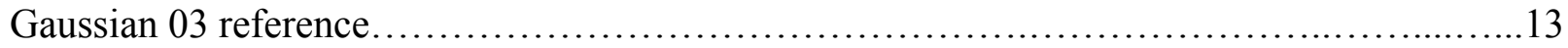




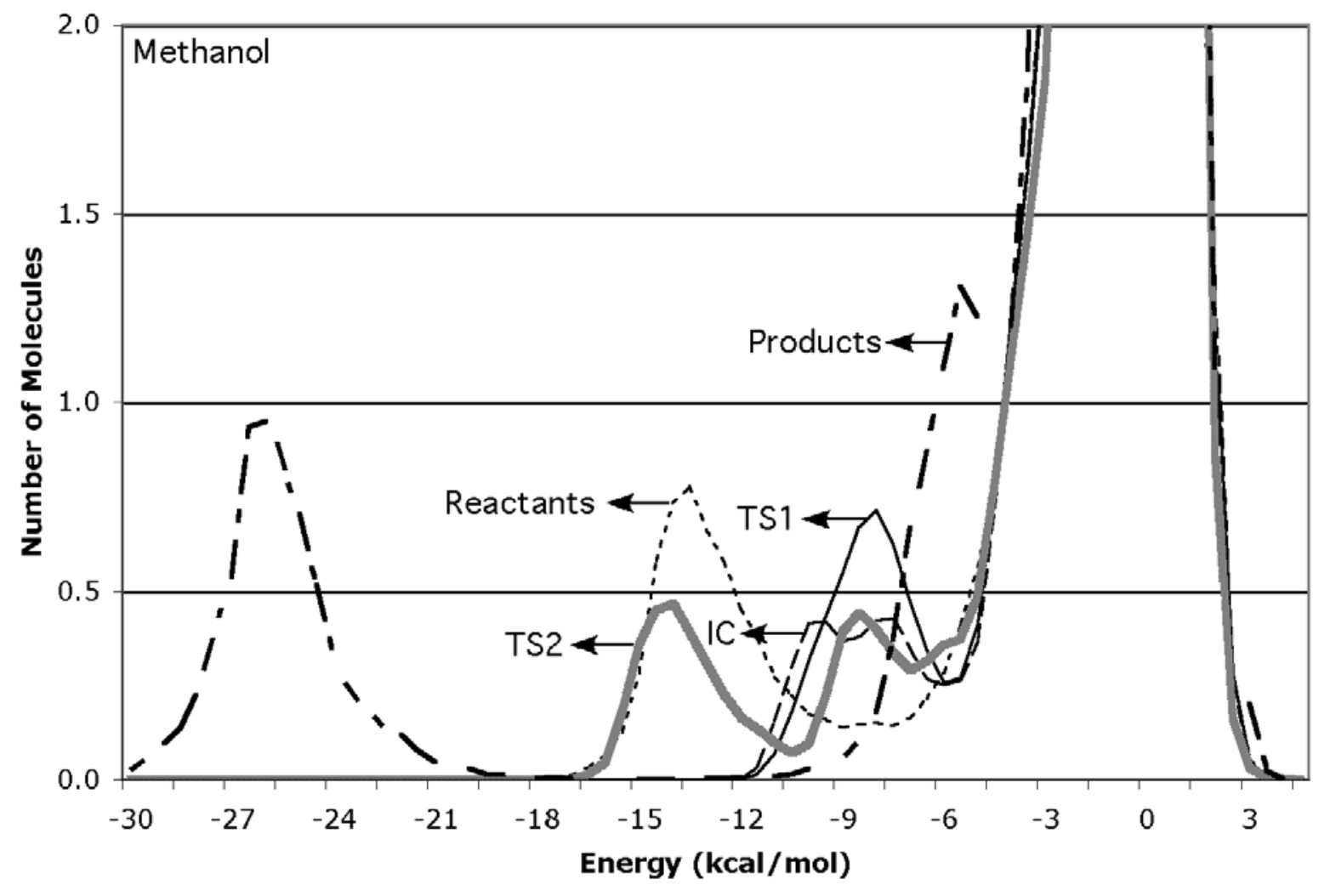

Figure S1. Solute-solvent energy pair distributions for the $\mathrm{S}_{\mathrm{N}} \mathrm{Ar}$ reaction in methanol for structures near the reactants, transition structures, intermediate complex and products. The plots show the number of molecules on the ordinate that interact with the solutes and their interaction energy in $\mathrm{kcal} / \mathrm{mol}$. 


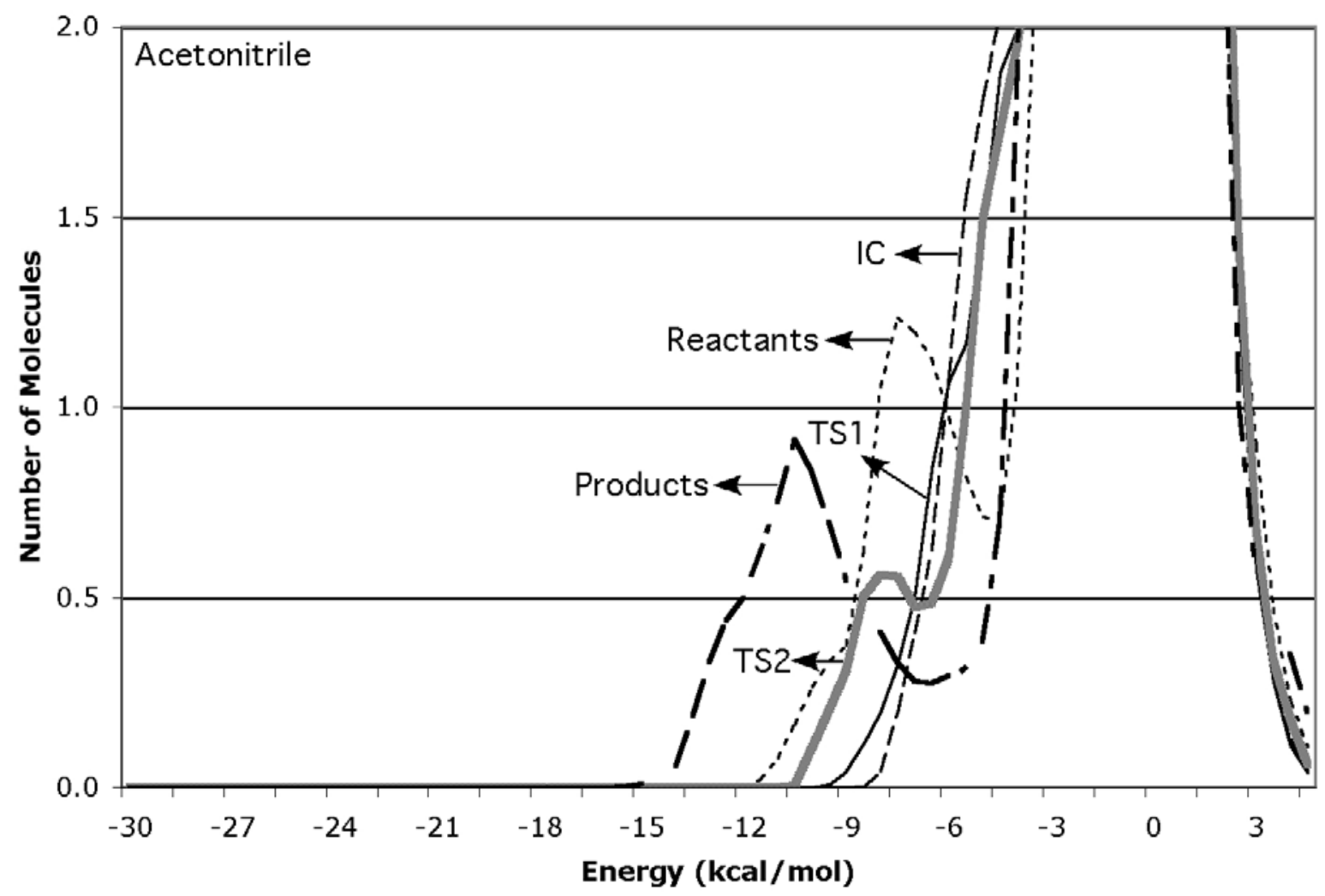

Figure S2. Solute-solvent energy pair distributions for the $\mathrm{S}_{\mathrm{N}} \mathrm{Ar}$ reaction in acetonitrile for structures near the reactants, transition structures, intermediate complex and products. The plots show the number of molecules on the ordinate that interact with the solutes and their interaction energy in $\mathrm{kcal} / \mathrm{mol}$. 


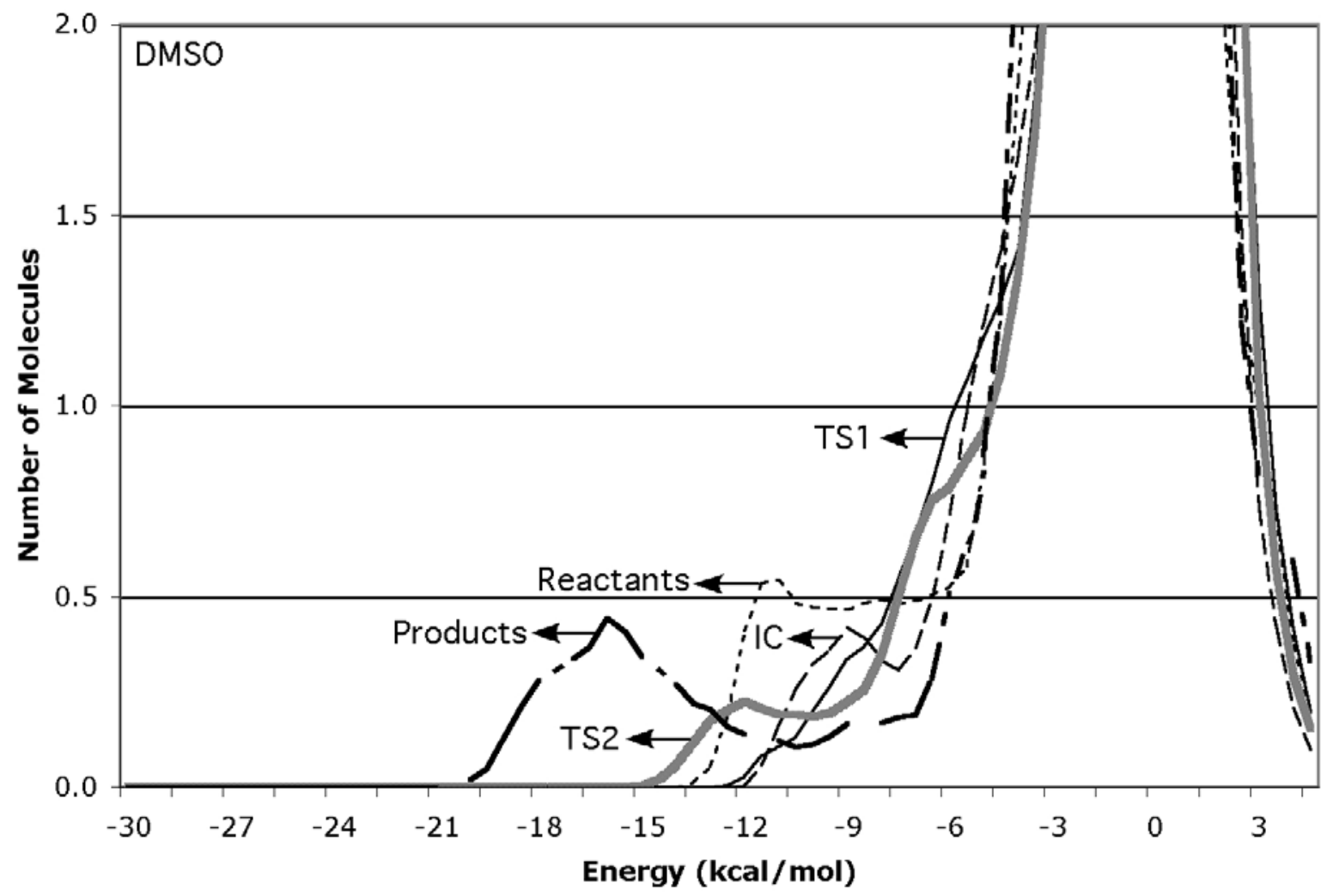

Figure S3. Solute-solvent energy pair distributions for the $S_{N} A r$ reaction in DMSO for structures near the reactants, transition structures, intermediate complex and products. The plots show the number of molecules on the ordinate that interact with the solutes and their interaction energy in $\mathrm{kcal} / \mathrm{mol}$. 
First TS in vacuum at B3LYP/6-31+G(d)

Freq $\backslash$ RB 3LYP \6-31+G (d) \C6H4F1N4O2 (1-) \ORLANDO \10-DeC

-2003\1\\\# B3LYP/6-31+G(D) FREQ GUESS=READ \TS1 of SnAr reaction betwe

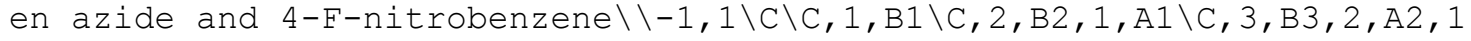
, D1, $0 \backslash \mathrm{C}, 4, \mathrm{~B} 4,3, \mathrm{~A} 3,2, \mathrm{D} 2,0 \backslash \mathrm{C}, 1, \mathrm{~B} 5,2, \mathrm{~A} 4,3, \mathrm{D} 3,0 \backslash \mathrm{H}, 2, \mathrm{~B} 6,1, \mathrm{~A} 5,6, \mathrm{D} 4,0 \backslash \mathrm{H}, 3, \mathrm{~B} 7$, $2, \mathrm{~A} 6,1, \mathrm{D} 5,0 \backslash \mathrm{H}, 5, \mathrm{~B} 8,4, \mathrm{~A} 7,3, \mathrm{D} 6,0 \backslash \mathrm{H}, 6, \mathrm{~B} 9,1, \mathrm{~A} 8,2, \mathrm{D} 7,0 \backslash \mathrm{N}, 1, \mathrm{~B} 10,2, \mathrm{~A} 9,3, \mathrm{D} 8,0 \backslash$ $0,11, \mathrm{~B} 11,1, \mathrm{~A} 10,2, \mathrm{D} 9,0 \backslash 0,11, \mathrm{~B} 12,1, \mathrm{~A} 11,2, \mathrm{D} 10,0 \backslash \mathrm{F}, 4, \mathrm{~B} 13,3, \mathrm{~A} 12,2, \mathrm{D} 11,0 \backslash \mathrm{N}, 4$

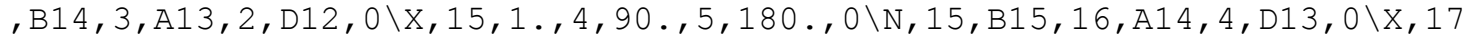
$, 1 ., 15,90 ., 16,0 ., 0 \backslash N, 17, B 16,18, A 15,15, \mathrm{D} 14,0 \backslash \backslash \mathrm{B} 1=1.4144 \backslash \mathrm{B} 2=1.3754 \backslash \mathrm{B} 3=1$. $434 \backslash B 4=1.4341 \backslash B 5=1.4144 \backslash B 6=1.0842 \backslash B 7=1.0855 \backslash B 8=1.0855 \backslash B 9=1.0842 \backslash B 10=1$. $4158 \backslash B 11=1.2535 \backslash B 12=1.2535 \backslash B 15=1.2091 \backslash B 16=1.1644 \backslash A 1=120.5204 \backslash A 2=120.59$ $32 \backslash A 3=117.8696 \backslash A 4=119.506 \backslash A 5=118.8033 \backslash A 6=121.359 \backslash A 7=118.0038 \backslash A 8=118.80$ $4 \backslash A 9=120.237 \backslash A 10=119.0958 \backslash A 11=119.0962 \backslash A 12=115.0356 \backslash A 13=106.9141 \backslash A 14=1$ $14.389 \backslash A 15=89.947 \backslash D 1=3.6948 \backslash D 2=-7.1611 \backslash D 3=0.0032 \backslash D 4=181.6101 \backslash D 5=181.26$ $91 \backslash D 6=-175.1804 \backslash D 7=178.3901 \backslash D 8=-181.6242 \backslash D 9=180.9856 \backslash D 10=0.6569 \backslash D 11=-1$ $47.9927 \backslash D 12=113.1479 \backslash D 13=114.0514 \backslash D 14=180.2794 \backslash B 13=1.3842 \backslash B 14=1.94 \backslash \backslash V e$ rsion=x86-Linux-G03RevB.03\State $=1-A \backslash H F=-700.2642635 \backslash$ RMSD $=7.039 e-09 \backslash R M$ $\mathrm{SF}=2.427 e-05 \backslash \mathrm{Dipole}=0.6063162,-1.1665887,0.3529919$

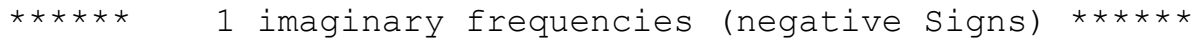

Diagonal vibrational polarizability:

$214.1610805 \quad 129.4472096 \quad 13.7355711$

Harmonic frequencies $\left(\mathrm{cm}^{*} *-1\right)$, IR intensities (KM/Mole), Raman scattering activities (A**4/AMU), depolarization ratios for plane and unpolarized incident light, reduced masses (AMU), force constants(mDyne/A), and normal coordinates:

\begin{tabular}{lrrr} 
& \multicolumn{1}{c}{1} & \multicolumn{1}{c}{2} & 3 \\
& \multicolumn{1}{c}{ A } & A & A \\
Frequencies -- & -254.4764 & 17.9605 & 53.8225 \\
Red. masses -- & 10.9973 & 8.5758 & 13.1591 \\
Frc consts -- & 0.4196 & 0.0016 & 0.0225 \\
IR Inten -- & 556.7799 & 0.0692 & 3.8019
\end{tabular}

Zero-point correction=

0.105190

(Hartree/Particle)

Thermal correction to Energy=

0.115994

Thermal correction to Enthalpy=

0.116938

Thermal correction to Gibbs Free Energy=

Sum of electronic and zero-point Energies=

0.066333

Sum of electronic and thermal Energies=

Sum of electronic and thermal Enthalpies=

$-700.159074$

$-700.148269$

$-700.147325$

Sum of electronic and thermal Free Energies=

$-700.197931$ 
Second TS in vacuum at B3LYP/6-31+G(d)

Freq $\backslash$ RB 3LYP \6-31+G (d) \C6H4F1N4O2 (1-) \ORLANDO \11-DeC

-2003\1\\\# B3LYP/6-31+G(D) FREQ GUESS=READ \TS2 of SnAr reaction betwe

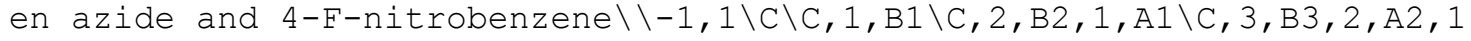
, D1, $0 \backslash \mathrm{C}, 4, \mathrm{~B} 4,3, \mathrm{~A} 3,2, \mathrm{D} 2,0 \backslash \mathrm{C}, 1, \mathrm{~B} 5,2, \mathrm{~A} 4,3, \mathrm{D} 3,0 \backslash \mathrm{H}, 2, \mathrm{~B} 6,1, \mathrm{~A} 5,6, \mathrm{D} 4,0 \backslash \mathrm{H}, 3, \mathrm{~B} 7$, $2, \mathrm{~A} 6,1, \mathrm{D} 5,0 \backslash \mathrm{H}, 5, \mathrm{~B} 8,4, \mathrm{~A} 7,3, \mathrm{D} 6,0 \backslash \mathrm{H}, 6, \mathrm{~B} 9,1, \mathrm{~A} 8,2, \mathrm{D} 7,0 \backslash \mathrm{N}, 1, \mathrm{~B} 10,2, \mathrm{~A} 9,3, \mathrm{D} 8,0 \backslash$ $0,11, \mathrm{~B} 11,1, \mathrm{~A} 10,2, \mathrm{D} 9,0 \backslash 0,11, \mathrm{~B} 12,1, \mathrm{~A} 11,2, \mathrm{D} 10,0 \backslash \mathrm{F}, 4, \mathrm{~B} 13,3, \mathrm{~A} 12,2, \mathrm{D} 11,0 \backslash \mathrm{N}, 4$

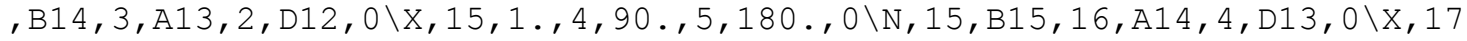
$, 1 ., 15,90.16,0 ., 0 \backslash \mathrm{N}, 17, \mathrm{~B} 16,18, \mathrm{~A} 15,15, \mathrm{D} 14,0 \backslash \backslash \mathrm{B} 1=1.4018 \backslash \mathrm{B} 2=1.3855 \backslash \mathrm{B} 3=1$. $409 \backslash B 4=1.4165 \backslash B 5=1.4106 \backslash B 6=1.0837 \backslash B 7=1.0852 \backslash B 8=1.0842 \backslash B 9=1.0834 \backslash B 10=1$. $4366 \backslash \mathrm{B} 11=1.2459 \backslash \mathrm{B} 12=1.2468 \backslash \mathrm{B} 15=1.2405 \backslash \mathrm{B} 16=1.1378 \backslash \mathrm{A} 1=119.5177 \backslash \mathrm{A} 2=120.61$ $77 \backslash A 3=119.1587 \backslash A 4=120.5403 \backslash A 5=119.3953 \backslash A 6=120.8026 \backslash A 7=119.2246 \backslash A 8=119$. $3072 \backslash A 9=119.6323 \backslash A 10=118.7227 \backslash A 11=118.912 \backslash A 12=112.8624 \backslash A 13=115.4491 \backslash A 1$ $4=134.6986 \backslash A 15=86.0149 \backslash D 1=-2.2251 \backslash D 2=3.3296 \backslash D 3=0.2848 \backslash D 4=179.6955 \backslash D 5=1$ $79.7594 \backslash D 6=-179.8648 \backslash D 7=179.7063 \backslash D 8=-178.4768 \backslash D 9=178.4947 \backslash D 10=-1.2324 \backslash$ $\mathrm{D} 11=-94.8856 \backslash \mathrm{D} 12=168.4371 \backslash \mathrm{D} 13=227.9944 \backslash \mathrm{D} 14=168.6636 \backslash \mathrm{B} 13=2.3149 \backslash \mathrm{B} 14=1.4$ $098 \backslash \backslash$ Version=x86-Linux-G03RevB.03\State=1-A \HF=-700.2622303 $\backslash$ RMSD=4.141 $e-09 \backslash \operatorname{RMSF}=5.399 e-05 \backslash \mathrm{Dipole}=-0.3201917,2.1863034,0.2318938$

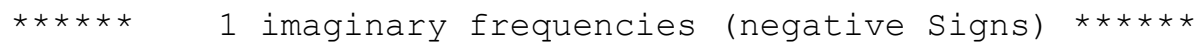

Diagonal vibrational polarizability:

253.4546450 $39.9853279 \quad 125.8354506$

Harmonic frequencies $\left(\mathrm{cm}^{*} *-1\right)$, IR intensities (KM/Mole), Raman scattering activities (A**4/AMU), depolarization ratios for plane and unpolarized incident light, reduced masses (AMU), force constants (mDyne/A), and normal coordinates:

\begin{tabular}{|c|c|c|c|c|}
\hline & & 1 & 2 & 3 \\
\hline & & A & A & A \\
\hline Frequencies & -- & -87.1123 & 55.8865 & 74.0992 \\
\hline Red. masses & -- & 9.1098 & 11.4926 & 11.7629 \\
\hline Frc consts & -- & 0.0407 & 0.0211 & 0.0381 \\
\hline IR Inten & -- & 51.5983 & 0.2463 & 4.3331 \\
\hline
\end{tabular}

Zero-point correction=

0.105955

(Hartree/Particle)

Thermal correction to Energy=

0.116760

Thermal correction to Enthalpy=

Thermal correction to Gibbs Free Energy=

Sum of electronic and zero-point Energies=

Sum of electronic and thermal Energies=

Sum of electronic and thermal Enthalpies=

Sum of electronic and thermal Free Energies=

0.117704

0.068216

$-700.156275$

$-700.145470$

$-700.144526$

$-700.194014$ 
IC in vacuum at $6-31+G(d)$

Freq $\backslash R B 3 L Y P \backslash 6-31+G(d) \backslash C 6 H 4 F 1 N 402$ (1-) \ORLANDO \08-Dec

-2003\1\\\# B3LYP/6-31+G(D) FREQ GUESS=READ \IC of SnAr reaction betwee

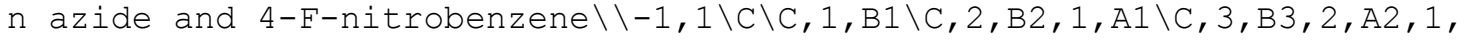
$\mathrm{D} 1,0 \backslash \mathrm{C}, 4, \mathrm{~B} 4,3, \mathrm{~A} 3,2, \mathrm{D} 2,0 \backslash \mathrm{C}, 1, \mathrm{~B} 5,2, \mathrm{~A} 4,3, \mathrm{D} 3,0 \backslash \mathrm{H}, 2, \mathrm{~B} 6,1, \mathrm{~A} 5,6, \mathrm{D} 4,0 \backslash \mathrm{H}, 3, \mathrm{~B} 7,2$ $, \mathrm{A} 6,1, \mathrm{D} 5,0 \backslash \mathrm{H}, 5, \mathrm{~B} 8,4, \mathrm{~A} 7,3, \mathrm{D} 6,0 \backslash \mathrm{H}, 6, \mathrm{~B} 9,1, \mathrm{~A} 8,2, \mathrm{D} 7,0 \backslash \mathrm{N}, 1, \mathrm{~B} 10,2, \mathrm{~A} 9,3, \mathrm{D} 8,0 \backslash 0$

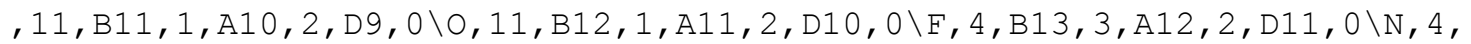
$\mathrm{B} 14,3, \mathrm{~A} 13,2, \mathrm{D} 12,0 \backslash \mathrm{X}, 15,1.4,90.5,180.0 \backslash \mathrm{N}, 15, \mathrm{~B} 15,16, \mathrm{~A} 14,4, \mathrm{D} 13,0 \backslash \mathrm{X}, 17$, $1 ., 15,90 ., 16,0 ., 0 \backslash N, 17, B 16,18, A 15,15, \mathrm{D} 14,0 \backslash \backslash \mathrm{B} 1=1.4203 \backslash \mathrm{B} 2=1.3666 \backslash \mathrm{B} 3=1.4$ $716 \backslash B 4=1.4696 \backslash B 5=1.4218 \backslash B 6=1.0848 \backslash B 7=1.0876 \backslash B 8=1.0864 \backslash B 9=1.0848 \backslash B 10=1$. $4003 \backslash B 11=1.2604 \backslash B 12=1.2605 \backslash B 13=1.5125 \backslash B 14=1.5045 \backslash B 15=1.2282 \backslash B 16=1.1472$ $\backslash A 1=121.2842 \backslash A 2=121.9622 \backslash A 3=114.3479 \backslash A 4=118.582 \backslash A 5=118.3846 \backslash A 6=120.434$ $9 \backslash A 7=116.7419 \backslash A 8=118.34 \backslash A 9=120.6636 \backslash A 10=119.4066 \backslash A 11=119.4307 \backslash A 12=107$. $8412 \backslash A 13=115.0272 \backslash A 14=26.6369 \backslash A 15=96.1808 \backslash D 1=-3.0417 \backslash D 2=7.6451 \backslash D 3=-1.7$ $458 \backslash D 4=177.4538 \backslash D 5=179.6785 \backslash D 6=-186.2637 \backslash D 7=182.707 \backslash D 8=-179.6114 \backslash D 9=17$ $9.1814 \backslash \mathrm{D} 10=-0.9275 \backslash \mathrm{D} 11=-113.6092 \backslash \mathrm{D} 12=135.4507 \backslash \mathrm{D} 13=180.7721 \backslash \mathrm{D} 14=178.964$ $4 \backslash \backslash$ Version=x86-Linux-G03RevB.03 State=1-A \HF=-700.2701656 RMSD=7.497e$09 \backslash \mathrm{RMSF}=2.412 \mathrm{e}-05 \backslash \mathrm{Dipole}=1.349193,0.401698,0.8118316$

$\begin{array}{ll}\text { Zero-point correction= } & 0.106054\end{array}$

(Hartree/Particle)

Thermal correction to Energy= 0.116981

Thermal correction to Enthalpy= 0.117925

Thermal correction to Gibbs Free Energy= 0.068097

Sum of electronic and zero-point Energies= $\quad-700.164112$

Sum of electronic and thermal Energies= $\quad-700.153185$

Sum of electronic and thermal Enthalpies= $\quad-700.152241$

Sum of electronic and thermal Free Energies= $\quad-700.202069$ 


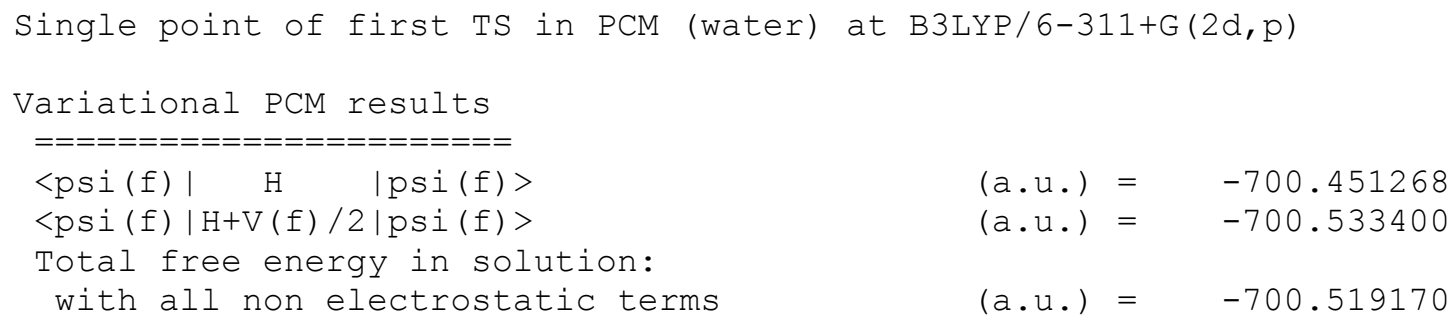

$==================$

$<\operatorname{psi}(\mathrm{f})|\mathrm{H} \quad| \operatorname{psi}(\mathrm{f})\rangle$

$(\mathrm{a} \cdot \mathrm{u})=$.

<psi(f) |H+V(f)/2|psi(f)>

$(\mathrm{a} \cdot \mathrm{u})=$.

Total free energy in solution:

with all non electrostatic terms

$(\mathrm{a} \cdot \mathrm{u})=$. 


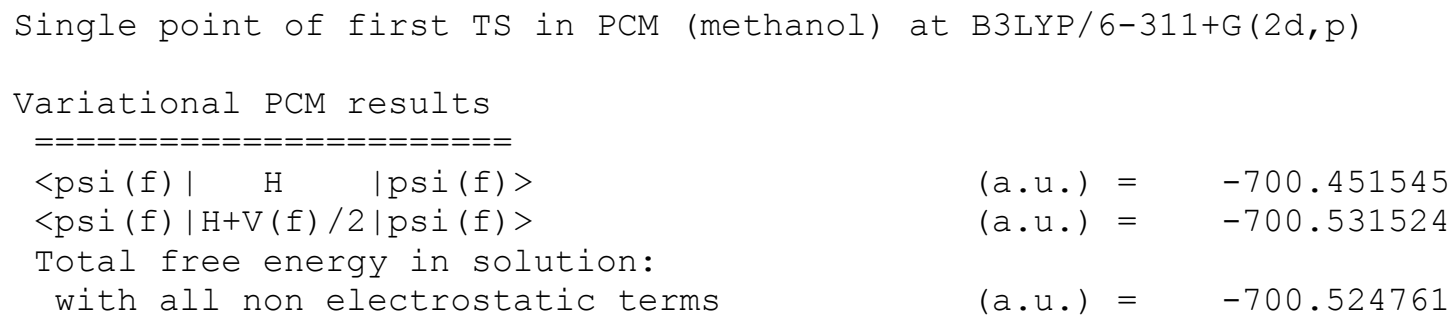

Single point of second TS in PCM (methanol) at B3LYP/6-311+G $(2 \mathrm{~d}, \mathrm{p})$

Variational PCM results

\begin{tabular}{|c|c|c|}
\hline$<\operatorname{psi}(f)|\quad H \quad| \operatorname{psi}(f)>$ & $(\mathrm{a} \cdot \mathrm{u} \cdot)=$ & -700.444630 \\
\hline$<\operatorname{psi}(f)|H+V(f) / 2| \operatorname{psi}(f)>$ & $(\mathrm{a} \cdot \mathrm{u} \cdot)=$ & -700.549768 \\
\hline $\begin{array}{l}\text { Total free energy in solution: } \\
\text { with all non electrostatic terms }\end{array}$ & $(\mathrm{a} \cdot \mathrm{u} \cdot)=$ & -700.5432 \\
\hline
\end{tabular}

Single point of IC in PCM (methanol) at B3LYP/6-311+G(2d,p)

Variational $\mathrm{PCM}$ results

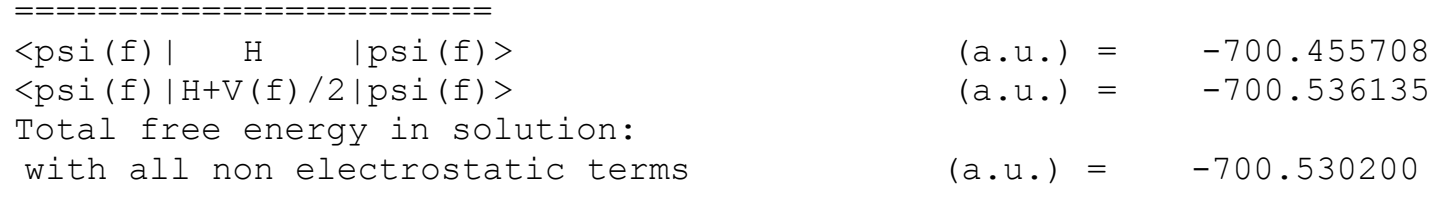




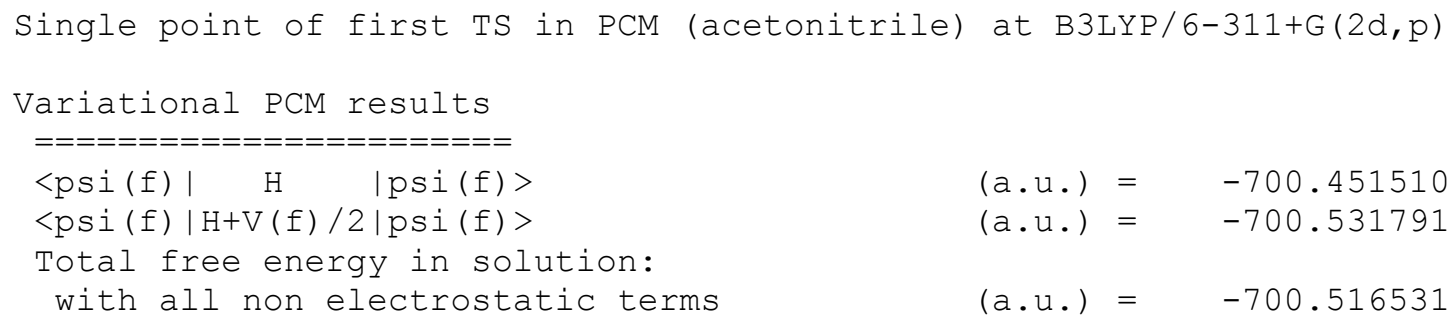

Single point of second TS in PCM (acetonitrile) at B3LYP/6-311+G (2d,p)

Variational PCM results

\begin{tabular}{|c|c|c|}
\hline$<\operatorname{psi}(f)|\quad H \quad| \operatorname{psi}(f)>$ & $(\mathrm{a} \cdot \mathrm{u} \cdot)=$ & -700.444645 \\
\hline$<\operatorname{psi}(f)|H+V(f) / 2| \operatorname{psi}(f)>$ & $(\mathrm{a} \cdot \mathrm{u} \cdot)=$ & -700.549789 \\
\hline $\begin{array}{l}\text { Total free energy in solution: } \\
\text { with all non electrostatic terms }\end{array}$ & $(\mathrm{a} \cdot \mathrm{u} \cdot)=$ & -700.534720 \\
\hline
\end{tabular}

Single point of IC in PCM (acetonitrile) at B3LYP/6-311+G (2d,p)

Variational $\mathrm{PCM}$ results

\begin{tabular}{|c|c|c|c|}
\hline$<\operatorname{psi}(f)|\quad H \quad| \operatorname{psi}(f)>$ & $(\mathrm{a} \cdot \mathrm{u} \cdot)$ & $=$ & -700.455687 \\
\hline$<\operatorname{psi}(f)|H+V(f) / 2| \operatorname{psi}(f)>$ & $(\mathrm{a} \cdot \mathrm{u} \cdot)$ & $=$ & -700.536351 \\
\hline
\end{tabular}




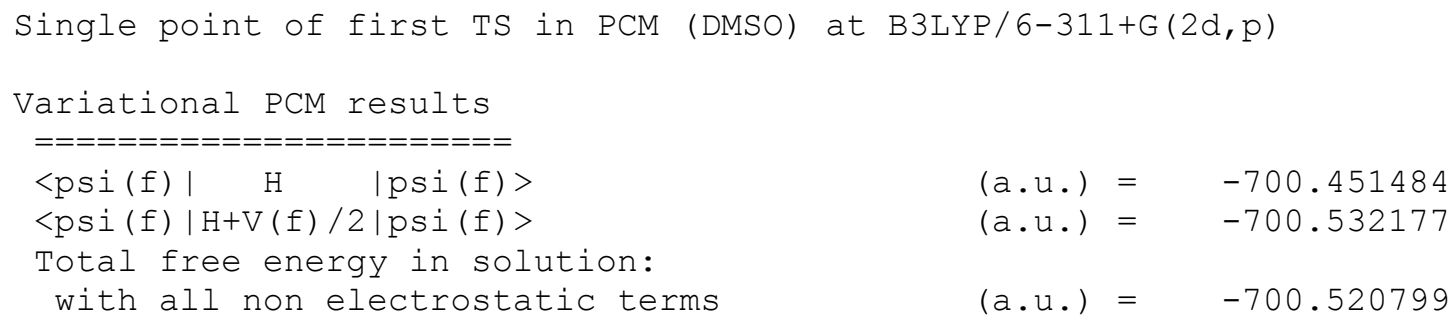


Gaussian 03, Revision B.03,

M. J. Frisch, G. W. Trucks, H. B. Schlegel, G. E. Scuseria,

M. A. Robb, J. R. Cheeseman, J. A. Montgomery, Jr., T. Vreven,

K. N. Kudin, J. C. Burant, J. M. Millam, S. S. Iyengar, J. Tomasi,

V. Barone, B. Mennucci, M. Cossi, G. Scalmani, N. Rega,

G. A. Petersson, H. Nakatsuji, M. Hada, M. Ehara, K. Toyota,

R. Fukuda, J. Hasegawa, M. Ishida, T. Nakajima, Y. Honda, O. Kitao,

H. Nakai, M. Klene, X. Li, J. E. Knox, H. P. Hratchian, J. B. Cross,

C. Adamo, J. Jaramillo, R. Gomperts, R. E. Stratmann, O. Yazyev,

A. J. Austin, R. Cammi, C. Pomelli, J. W. Ochterski, P. Y. Ayala,

K. Morokuma, G. A. Voth, P. Salvador, J. J. Dannenberg,

V. G. Zakrzewski, S. Dapprich, A. D. Daniels, M. C. Strain,

O. Farkas, D. K. Malick, A. D. Rabuck, K. Raghavachari,

J. B. Foresman, J. V. Ortiz, Q. Cui, A. G. Baboul, S. Clifford,

J. Cioslowski, B. B. Stefanov, G. Liu, A. Liashenko, P. Piskorz,

I. Komaromi, R. L. Martin, D. J. Fox, T. Keith, M. A. Al-Laham,

C. Y. Peng, A. Nanayakkara, M. Challacombe, P. M. W. Gill,

B. Johnson, W. Chen, M. W. Wong, C. Gonzalez, and J. A. Pople, Gaussian, Inc., Pittsburgh PA, 2003. 